\title{
Síndrome de burnout entre médicos plantonistas de unidades de terapia intensiva
}

\author{
Burnout syndrome among intensive care unit physicians \\ Gabriela Lopes Carvalho Marques', Flávia Lopes Carvalho², Sandra Fortes², Hamilton Raposo Miranda Filho', \\ Gilberto Sousa Alves 3
}

\section{RESUMO}

Objetivos: Estimar a prevalência da síndrome de burnout (SB) e fatores associados entre médicos plantonistas de unidades de terapia intensiva (UTI) de São Luís-MA. Métodos: Participaram 60 médicos plantonistas de seis UTIs de São Luís-MA, investigados em setembro a dezembro de 2012, por meio de um questionário sociodemográfico abrangendo características condições de trabalho, fatores estressantes e do Maslach Burnout Inventory (MBI). Foram realizadas estatística descritiva e análise dos fatores associados à SB por meio do teste de Qui-quadrado e Razão de Prevalência. Resultados: Mulheres $(n=38)$, entre 30 e 39 anos, formadas há 10 anos ou menos, atuando há cinco anos ou menos em UTI, em dois hospitais, cuidando de seis a dez pacientes/plantão e com elevada carga horária, foram o público prevalente. Os principais fatores estressantes foram ruídos excessivos e possibilidade de complicação no atendimento. A prevalência do burnout com escore elevado (pontuações maiores ou iguais a 27 na exaustão emocional e 13 na despersonalização e menores que 31 na redução da realização profissional) foi de 13,3\% em todas as dimensões e de 50\% em pelo menos uma delas; níveis elevados de exaustão emocional estiveram presentes em 35\% da amostra, seguidos pela baixa realização profissional (25\%) e despersonalização (6,7\%). Con-

\section{Palavras-chave}

Condições de trabalho, estresse, esgotamento profissional, síndrome de burnout, unidades de terapia intensiva. clusões: A prevalência do burnout foi elevada, sendo mais frequente em mulheres, na UTI adulto, nos sujeitos que trabalhavam em dois ou mais hospitais e que assistiam mais de dez pacientes por plantão.

\section{ABSTRACT}

Objectives: To estimate the prevalence of burnout syndrome (SB) and associated factors among attending physicians of intensive care units (ICUs) of São Luís-MA. Methods: Participants included 60 physicians on 6 MTIs from the metropolitan area of São Luís-MA investigated in September to December of 2012, using a self-administered questionnaire developed by the authors, covering sociodemographic characteristics, working conditions, stressors and the Maslach Burnout Inventory (MBI). Descriptive statistics and analysis of factors associated with SB through the Chi-square test and Prevalence Ratio. Results: Most are women ( $n=$

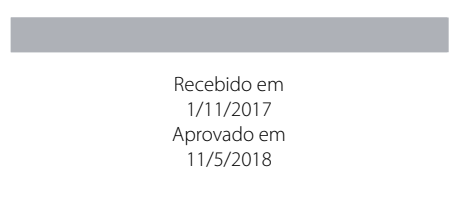

DOI: $10.1590 / 0047-2085000000202$
1 Secretaria Estadual de Saúde do Maranhão, Maranhão, MA, Brasil. 2 Universidade do Estado do Rio de Janeiro (UERJ), Rio de Janeiro, RJ, Brasil. 3 Universidade Federal do Ceará (UFC), Fortaleza, CE, Brasil.
Endereço para correspondência: Gilberto Sousa Alves Universidade Federal do Ceará (UFC), Fortaleza, CE, Brasil E-mail: gsalves123@hotmail.com 


\section{Keywords}

Working conditions, stress, exhaustion professional, burnout syndrome, intensive care units.
38), between 30-39 years, formed 10 years ago or less, operating for five years or less in the ICU, in two hospitals, caring for six to ten patients/duty and high workload. The main stress factors were excessive noise and possibility of complications in attendance. The prevalence of burnout with a high score (scores greater than or equal to 27 in emotional exhaustion and 13 in depersonalization and less than 31 in reducing professional achievement) in one of the three dimensions was 50\%, high scores in all three dimensions was 13.3\%, and high levels in the dimensions analyzed separately in $35 \%$ of emotional exhaustion, depersonalization $6.7 \%$ in and 25\% reduction in job satisfaction. Conclusions: The prevalence of burnout syndrome was high, being more common in women, in the adult ICU, work in more than two hospitals and attend more than ten patients per shift.

\section{INTRODUÇÃO}

O trabalho é uma atividade que pode ocupar grande parcela do tempo de cada indivíduo e do seu convívio em sociedade1, e nem sempre possibilita a realização profissional, podendo, ao contrário, causar problemas desde insatisfação até exaustão².

Nos últimos anos, a relação entre estresse ocupacional e saúde mental dos trabalhadores tem sido amplamente discutida ${ }^{3-6}$, principalmente em decorrência dos níveis alarmantes de incapacidade temporária, absenteísmo, aposentadorias precoces e riscos à saúde associados à atividade profissional ${ }^{4}$.

Entre as consequências psíquicas geradas por problemas ocupacionais, estão a síndrome de burnout, um termo utilizado para explicar o sofrimento do homem em seu ambiente de trabalho, associado a perda de motivação e alto grau de insatisfação, decorrentes dessa exaustão?.

A síndrome de burnout ou síndrome da estafa profissional constitui um quadro bem definido, caracterizado por exaustão emocional, despersonalização e redução da realização pessoal ${ }^{8}$. A exaustão emocional representa o esgotamento dos recursos emocionais do indivíduo, decorrendo principalmente da sobrecarga e do conflito pessoal nas relações interpessoais, sendo considerado o traço inicial da síndrome ${ }^{8,9}$. A despersonalização é caracterizada pela insensibilidade emocional do profissional, que passa a tratar os paciente e colegas como objetos ${ }^{8}$. Trata-se de um aspecto fundamental para caracterizar a síndrome de burnout, já que suas outras características podem ser encontradas nos quadros depressivos em gera|8,9. Por fim, a redução da realização pessoal (ou sentimento de incompetência ou ineficiência) revela uma autoavaliação negativa associada a insatisfação e infelicidade com o trabalho $0^{8,9}$

São vários os sintomas atribuídos à síndrome, sendo eles divididos em quatro categorias: físicos (fadiga, distúrbios do sono, dores musculares, cefaleias), psíquicos (falta de aten- ção e/ou concentração, sentimento de solidão, baixa autoestima, labilidade emocional), comportamentais (negligência, irritabilidade, comportamento de autorrisco) e defensivos (tendência ao isolamento, perda de interesse pelo trabalho, sentimento de incompetência) ${ }^{9}$.

A duração do burnout pode variar de anos até décadas ${ }^{10}$. Sua ocorrência costuma ainda ser insidiosa e cumulativa, com incremento progressivo na gravidade, podendo não ser percebida pelo indivíduo, que nesses casos se recusa a acreditar estar acontecendo algo de errado com ele ${ }^{10,11}$.

Entre as variáveis responsáveis pelo desencadeamento da síndrome de burnout, encontramos: características pessoais (idade, nível educacional, estado civil), características do trabaIho (tempo de profissão, tipo de ocupação, relação com clientes/colegas), características organizacionais (ambiente físico, mudanças organizacionais, normas institucionais) e características sociais (suporte social e familiar, cultura e prestígio)9,12.

Burnout é um tipo de estresse ocupacional que acomete profissionais envolvidos com qualquer tipo de cuidado, sendo os trabalhadores que atendem ou assistem pessoas em situação de risco ou de extrema responsabilidade normalmente os mais acometidos $8,12,13$.

Entre os fatores ambientais mais relacionados ao burnout no ambiente da unidade de terapia intensiva (UTI), estão a convivência recorrente com a situação de morte, o contato com as famílias e o medo de ser responsabilizado pelo fracasso terapêutico dos pacientes ${ }^{14-16}$. Outros estudos citam ainda características do ambiente físico dessas unidades, como quartos fechados, com condições e ritmos de trabalho extenuantes, rotinas exigentes, questões éticas em que cabem decisões frequentes e difíceis, imprevisibilidade e carga horária de trabalho excessiva ${ }^{14,17-20}$.

A prevalência da síndrome de burnout ainda é incerta, mas dados sugerem que acomete um número significativo de indivíduos, variando de 4\% - quando três dimensões estão presentes - a 85,7\%, quando apenas uma dimensão está acometida ${ }^{1,18,19,20 .}$ 
Portanto, o burnout pode ser considerado um grande problema no mundo profissional da atualidade ${ }^{1,21}$, fazendo uma relação direta do homem contemporâneo e as exigências do trabalho em que está inserido.

Este trabalho tem como objetivo descrever as características sociodemográficas e as condições de trabalho dos médicos plantonistas das UTIs, listar os principais fatores estressantes em UTIs, verificar a presença da síndrome de burnout na população referida e associar os dados sociodemográficos e as condições de trabalho com a síndrome.

\section{MÉTODOS}

Após aprovação pelo Comitê de Ética em Pesquisa da Universidade Ceuma, foi realizado um estudo observacional, descritivo e transversal, entre setembro e dezembro de 2012, abrangendo os médicos plantonistas das UTIs de seis hospitais em São Luís-MA, sendo três UTIs adulto e três UTIs neonatal. Foram incluídos os médicos com Residência Médica e/ou Programa de Especialização em Medicina Intensiva e/ ou experiência de, no mínimo, um ano, desde que concordassem em participar da pesquisa por meio da assinatura do Termo de Consentimento Livre e Esclarecido (TCLE), conforme a Resolução no 196/96, do Conselho Nacional de Saúde e suas complementares.

No município existem 12 UTIs, sendo quatro unidades especializadas no atendimento neonatal e oito unidades para tratamentos intensivos em adultos. Após liberação pela direção das unidades hospitalares das UTIs, os dados foram coletados em três UTIs neonatais e três UTIs adulto.

O cálculo amostral foi realizado com base nos resultados de estudos da prevalência da síndrome de burnout entre profissionais de saúde ${ }^{18,22}$, quando se aplicou o poder do teste de $85 \%$ e o nível alfa de significância de $5 \%$ e se chegou ao tamanho amostral mínimo de 50 médicos, por meio do Programa Biostat ${ }^{\oplus}$. Considerando possíveis perdas, a presente pesquisa foi idealizada com a amostra de 60 médicos plantonistas em UTIs, devido ao acréscimo de 20\% na amostra.

Para a coleta de dados, foi utilizado um questionário sociodemográfico elaborado pelos próprios autores, respondido pelos participantes, após esclarecimento e consentimento pelo TCLE.

O questionário apresenta três blocos de questões:

1. Identificação geral (Questionário Sociodemográfico): destinado a determinar o perfil dos indivíduos quanto às características sociodemográficas e condições de trabalho;

2. Fatores estressantes presentes em UTI;

3. Avaliação do nível de burnout por meio de perguntas baseadas no Maslach Burnout Inventory - Human Services Sorvem (MBI-HSS), em sua versão brasileira, traduzido e validado por Carlotto e Câmara ${ }^{23}$.
O Questionário MBI é composto por 22 afirmações sobre sentimentos e atitudes que englobam três dimensões fundamentais da síndrome, divididos em três escalas de sete pontos, que variam de 0 a 6 . Dessa maneira, foram descritas, de forma independente, cada uma das dimensões que caracterizam a estafa profissional.

A exaustão profissional será avaliada por nove itens, a despersonalização, por cinco e a realização pessoal, por oito. As notas de corte utilizadas serão as empregadas no estudo de Maslach ${ }^{13}$. Para exaustão emocional, uma pontuação maior ou igual a 27 indica alto nível, de 17 a 26, nível moderado e menor que 16, nível baixo. Para despersonalização, pontuações iguais ou maiores que 13 indicam alto nível, de 7 a 12, nível moderado e menores de 6, nível baixo. A pontuação relacionada à redução da realização profissional vai em direção oposta às outras, uma vez que pontuações de 0 a 31 indicam alto nível, de 32 a 38, nível moderado e maior ou igual a 39, nível baixo.

Descrevemos os resultados interpretados pelo questionário de Maslach, segundo os critérios de Ramirez et al..$^{24}$ e os critérios de Grunfeld et al. ${ }^{25}$; os primeiros definem burnout pela presença das três dimensões em níveis altos, enquanto os segundos aceitam apenas uma dimensão, independentemente de qual seja, para fazer o diagnóstico de síndrome.

A análise dos dados foi feita por meio do software Biostat ${ }^{\oplus}$. Foram utilizados os parâmetros da estatística descritiva adotando-se cálculos de frequências simples e relativas. Realizou-se análise da associação entre as variáveis sociodemográficas e condições de trabalho com o resultado do $\mathrm{MBI}$, segundo os critérios de Grunfeld et al.24, por meio de testes de associação (teste de Qui-quadrado e Razão de Prevalência). Em todos os testes, o nível de significância aplicado foi de $5 \%$, ou seja, foi considerado significativo quando $p<0,05$.

\section{RESULTADOS}

Dos 60 médicos plantonistas das UTIs, a maioria pertence ao sexo feminino, entre 30 e 39 anos, casadas, formadas há 10 anos ou menos, e as principais especialidades foram Neonatologia, Pediatria e Medicina Intensiva (Tabela 1).

Quanto às condições de trabalho, 30 médicos trabalham nas UTIs adulto e os outros 30, nas UTIs neonatal; atuam na UTI entre um e cinco anos, trabalham em dois hospitais, assistindo em média seis a dez pacientes por plantão, com carga de trabalho semanal entre 20 e 40 horas, e a maioria tem como carga horária habitual do seu turno 12 horas (Tabela 2).

Os médicos apontaram como os principais fatores estressantes em UTI os ruídos excessivos e a possibilidade de complicações no atendimento aos pacientes internados (Tabela 3). 
Tabela 1. Características sociodemográficas dos médicos plantonistas das unidades de terapia intensiva em São Luís-MA, 2012

\begin{tabular}{|c|c|c|}
\hline Variáveis & Frequência $(n=60)$ & Percentual (100\%) \\
\hline \multicolumn{3}{|l|}{ Gênero } \\
\hline Masculino & 22 & 36,7 \\
\hline Feminino & 38 & 63,3 \\
\hline \multicolumn{3}{|l|}{ Idade } \\
\hline 23-29 anos & 11 & 18,3 \\
\hline 30-39 anos & 28 & 46,7 \\
\hline 40 anos ou mais & 21 & 35,0 \\
\hline \multicolumn{3}{|l|}{ Estado civil } \\
\hline Solteiro & 16 & 26,7 \\
\hline Casado & 40 & 66,7 \\
\hline Divorciado & 04 & 06,7 \\
\hline \multicolumn{3}{|l|}{ Tempo de formado } \\
\hline$\leq 10$ anos & 46 & 76,7 \\
\hline 11 a 20 anos & 11 & 18,3 \\
\hline$\geq 21$ anos & 03 & 05,0 \\
\hline \multicolumn{3}{|l|}{ Especialidade médica } \\
\hline Clínica Geral & 08 & 13,3 \\
\hline Clínica Médica & 06 & 10,0 \\
\hline Medicina Intensiva & 10 & 16,7 \\
\hline Neonatologia & 15 & 25,0 \\
\hline Pediatria & 14 & 23,3 \\
\hline Outros & 07 & 11,7 \\
\hline
\end{tabular}

Em relação à síndrome de burnout, a prevalência de escore alto em uma das três dimensões do MBI, segundo os critérios de Grunfeld et al., foi de 50\%; a prevalência de escore alto nas três dimensões, de acordo os critérios de Ramirez et al., foi de $13,3 \%$, e a prevalência de escore alto em cada uma das três dimensões analisadas separadamente foi de 35\% de exaustão emocional, 6,7\% de despersonalização e 25\% de redução da realização profissional (Tabela 4).

A síndrome de burnout nas UTIs estudadas foi mais prevalente no sexo feminino, com menos de 40 anos, casadas, formadas há 10 anos ou menos e que possuíam especialidades médicas em saúde da criança (Tabela 5).

A prevalência de burnout foi mais elevada nas UTIs adulto, onde os profissionais trabalham em dois ou mais hospitais, assistem mais de 10 pacientes por plantão, com carga horária semanal menor que 60 horas, porém com turno maior ou igual a 12 horas (Tabela 6).

Ao relacionarmos as características sociodemográficas e as condições de trabalho com a síndrome de burnout foram encontradas associações estatisticamente significativas entre a síndrome e o sexo feminino $(p=0,0304)$, trabalho em UTI adulto $(p=0,0004)$, trabalhar em mais de dois hospitais $(p=0,0095)$ e número de pacientes maior que 10 por plantão $(p=0,0021)$ (Tabelas 5 e 6$)$.
Tabela 2. Condições de trabalho dos médicos plantonistas das unidades de terapia intensiva em São Luís-MA, 2012

\begin{tabular}{|c|c|c|}
\hline Variáveis & Frequência ( $n=60)$ & Percentual (100\%) \\
\hline \multicolumn{3}{|l|}{ Tipo de UTI } \\
\hline Adulto & 30 & 50,0 \\
\hline Neonatal & 30 & 50,0 \\
\hline \multicolumn{3}{|l|}{ Tempo de trabalho em UTI } \\
\hline 1 a 5 anos & 36 & 60,0 \\
\hline 6 a 10 anos & 10 & 16,7 \\
\hline 11 a 15 anos & 07 & 11,7 \\
\hline$\geq 16$ anos & 07 & 11,7 \\
\hline \multicolumn{3}{|l|}{ Quantidade de hospitais } \\
\hline 1 & 12 & 20,0 \\
\hline 2 & 22 & 36,7 \\
\hline 3 & 20 & 33,3 \\
\hline 4 ou mais & 06 & 10,0 \\
\hline \multicolumn{3}{|l|}{ Número de pacientes } \\
\hline 6 a 10 pacientes & 34 & 56,7 \\
\hline 11 a 15 pacientes & 17 & 28,3 \\
\hline$\geq 16$ pacientes & 09 & 15,0 \\
\hline \multicolumn{3}{|l|}{ Carga horária semanal } \\
\hline 20 a 40 horas semanais & 24 & 40,0 \\
\hline 41 a 60 horas semanais & 17 & 28,3 \\
\hline 61 a 80 horas semanais & 10 & 16,7 \\
\hline$\geq 81$ horas semanais & 09 & 15,0 \\
\hline \multicolumn{3}{|l|}{ Carga horária do turno } \\
\hline 06 horas & 18 & 30,0 \\
\hline 12 horas & 40 & 66,7 \\
\hline 24 horas & 02 & 03,3 \\
\hline
\end{tabular}

UTl: unidade de terapia intensiva.

Tabela 3. Fatores estressantes em unidades de terapia intensiva de acordo com os médicos plantonistas em São Luís-MA, 2012

\begin{tabular}{lcc}
\hline \multicolumn{1}{c}{ Fatores estressantes em UTI } & Frequência ( $\mathbf{n}=60)$ & Percentual (100\%) \\
\hline Ruídos excessivos na UTI & 45 & 75,0 \\
$\begin{array}{l}\text { Possibilidade de complicações no } \\
\text { atendimento }\end{array}$ & 43 & 71,7 \\
Problemas administrativos & 41 & 68,3 \\
Comprometimento da equipe multidisciplinar & 41 & 68,3 \\
Falta de recursos materiais & 39 & 65,0 \\
Lidar com sofrimento e morte & 38 & 63,3 \\
Lidar com diversas questões & 37 & 61,7 \\
simultaneamente & & \\
Quantidade de pacientes por médico & 34 & 56,7 \\
Ritmo acelerado das atividades profissionais & 32 & 53,3 \\
Cuidar do paciente terminal & 20 & 33,3 \\
Pressão para dar alta aos pacientes & 20 & 33,3 \\
Relacionamento com a equipe & 13 & 21,7 \\
multidisciplinar & & \\
\hline
\end{tabular}

UTI: unidade de terapia intensiva. 
Tabela 4. Avaliação do Maslach Burnout Inventory segundo diferentes critérios em médicos plantonistas das unidades de terapia intensiva em São Luís-MA, 2012

\begin{tabular}{|c|c|c|}
\hline \multirow{2}{*}{ Critérios } & \multicolumn{2}{|c|}{ Resultados } \\
\hline & Frequência $(n=60)$ & Percentual (100\%) \\
\hline \multicolumn{3}{|l|}{ Dimensões isoladas } \\
\hline \multicolumn{3}{|l|}{ Exaustão emocional } \\
\hline Baixo & 20 & 33,3 \\
\hline Moderado & 19 & 31,7 \\
\hline Alto & 21 & 35,0 \\
\hline \multicolumn{3}{|l|}{ Despersonalização } \\
\hline Baixo & 40 & 66,7 \\
\hline Moderado & 16 & 26,7 \\
\hline Alto & 04 & 06,7 \\
\hline \multicolumn{3}{|l|}{ Redução da realização pessoal } \\
\hline Baixo & 27 & 45,0 \\
\hline Moderado & 18 & 30,0 \\
\hline Alto & 15 & 25,0 \\
\hline \multicolumn{3}{|l|}{ Grunfeld et al. } \\
\hline \multicolumn{3}{|l|}{ Presença de: } \\
\hline \multicolumn{3}{|l|}{ Exaustão emocional em nível alto $0 \mathrm{U}$} \\
\hline \multicolumn{3}{|l|}{ Despersonalização em nível alto $0 U$} \\
\hline Redução da realização profissional em nível alto & 30 & 50,0 \\
\hline \multicolumn{3}{|l|}{ Ramirez et al. } \\
\hline \multicolumn{3}{|l|}{ Presença de: } \\
\hline \multicolumn{3}{|l|}{ Exaustão emocional em nível alto $\mathrm{E}$} \\
\hline \multicolumn{3}{|l|}{ Despersonalização em nível alto E } \\
\hline Redução da realização profissional em nível alto & 01 & 13,3 \\
\hline
\end{tabular}

Tabela 5. Associação entre as características sociodemográficas e a síndrome de burnout em médicos plantonistas das unidades de terapia intensiva em São Luís-MA, 2012

\begin{tabular}{lcccccc}
\hline \multirow{2}{*}{ Variáveis } & \multicolumn{6}{c}{ Sindrome de burnout } \\
\cline { 2 - 7 } & Total & Sim & Não & $R P$ & $p$ & IC95\% \\
\hline Gênero & 22 & 7 & 15 & 0,53 & $\mathbf{0 , 0 3 0 4}$ & $0,27-1,02$ \\
$\quad$ Masculino & 38 & 23 & 15 & & & \\
$\quad$ Feminino & & & & & & \\
Idade & 39 & 18 & 21 & 0,81 & 0,2941 & $0,48-1,33$ \\
$\leq 40$ anos & 21 & 12 & 9 & & & \\
$>40$ anos & & & & & & \\
Estado civil & 20 & 12 & 8 & 1,33 & 0,2057 & $0,81-2,19$ \\
$\quad$ Companheiro instável & 40 & 18 & 22 & & & \\
$\quad$ Companheiro estável & & & & & & \\
Tempo de formado & & & & & & \\
$\quad \leq 10$ anos & 46 & 22 & 24 & 0,84 & 0,3801 & $0,49-1,44$ \\
$>11$ anos & 14 & 8 & 6 & & & \\
Especialidade médica & & & & & & \\
$\quad$ Saúde do Adulto & 31 & 12 & 19 & 0,62 & 0,0606 & $0,37-1,06$ \\
$\quad$ Saúde da Criança & 29 & 18 & 11 & & & \\
\hline
\end{tabular}

Tabela 6. Associação entre as condições de trabalho e a síndrome de burnout em médicos plantonistas das unidades de terapia intensiva em São Luís-MA, 2012

\begin{tabular}{|c|c|c|c|c|c|c|}
\hline \multirow{2}{*}{ Variáveis } & \multicolumn{6}{|c|}{ Síndrome de burnout } \\
\hline & Total & Sim & Não & $R P$ & $p$ & IC $95 \%$ \\
\hline \multicolumn{7}{|l|}{ Tipo de UTI } \\
\hline Adulto & 30 & 22 & 8 & 2,75 & 0,0004 & $1,46-5,17$ \\
\hline Neonatal & 30 & 8 & 22 & & & \\
\hline \multicolumn{7}{|l|}{ Tempo em UTI } \\
\hline$\leq 10$ anos & 46 & 21 & 25 & 0,71 & 0,1799 & $0,43-1,17$ \\
\hline$>11$ anos & 14 & 9 & 5 & & & \\
\hline \multicolumn{7}{|l|}{$\begin{array}{l}\text { Quantidade de } \\
\text { hospitais }\end{array}$} \\
\hline$\leq 2$ & 34 & 12 & 22 & 0,51 & 0,0095 & $0,30-0,86$ \\
\hline$>2$ & 26 & 18 & & & & \\
\hline \multicolumn{7}{|l|}{$\begin{array}{l}\text { Número de } \\
\text { pacientes }\end{array}$} \\
\hline$\leq 10$ pacientes & 34 & 11 & 23 & 0,44 & 0,0021 & $0,26-0,76$ \\
\hline$>10$ pacientes & 26 & 19 & 7 & & & \\
\hline \multicolumn{7}{|l|}{$\begin{array}{l}\text { Carga horária } \\
\text { semanal }\end{array}$} \\
\hline$\leq 60 \mathrm{horas} / \mathrm{sem}$ & 41 & 22 & 19 & 1,27 & 0,2894 & $0,70-2,32$ \\
\hline$>60$ horas/sem & 19 & 8 & 11 & & & \\
\hline \multicolumn{7}{|l|}{$\begin{array}{l}\text { Carga horária do } \\
\text { turno }\end{array}$} \\
\hline$<12$ horas & 18 & 9 & 9 & 1 & 0,3891 & $0,58-1,74$ \\
\hline$\geq 12$ horas & 42 & 21 & 21 & & & \\
\hline
\end{tabular}

\section{DISCUSSÃO}

O perfil dos médicos plantonistas das UTIs em São Luís-MA é de uma população jovem, predominantemente feminina, casada, com menos de dez anos de formada, tendo como principais especialidades a Neonatologia, seguida da Pediatria e Medicina Intensiva, trabalhando há menos de cinco anos na UTI, em cerca de dois hospitais, cuidando de seis a dez pacientes por plantão e com elevada carga horária de trabalho.

Quanto às características sociodemográficas e condições de trabalho, os resultados definem um perfil que se assemelha ao encontrado na população médica nacional|3,14,26, exceto pela predominância feminina, possivelmente explicada pela maior procura das especialidades pediátricas por médicas, dados confirmados ao comparar nossos resultados com o estudo de Lacerda et al..$^{27}$.

Em relação aos fatores estressantes em UTI, os plantonistas avaliados apontaram como os principais os ruídos excessivos e a possibilidade de complicações no atendimento aos pacientes internados, resultados esses semelhantes aos encontrados na literatura ${ }^{14,15,28}$. 
As UTIs são um ambiente com elevado estresse e ansiedade associados, motivados pelo relacionamento interpessoal, emoções intensas causadas pela exposição constante ao risco de morte, pela frequente oscilação entre sucesso e fracasso e pelas exigências impostas à equipe, e todos esses estímulos levam ao surgimento de sentimentos como inadequação, insegurança e impotência, capazes de influenciar de forma negativa a qualidade de vida ${ }^{3}$, predispondo à síndrome de burnout.

A prevalência da síndrome de burnout encontrada foi alta (50\%, segundo os critérios de Grunfeld et al., e 13,3\%, se utilizarmos os critérios de Ramirez et al.). No entanto, na literatura essa prevalência varia muito entre os estudos, dependendo da população estudada e dos valores conceituais utilizados como referência.

Utilizando critérios semelhantes, Barros et al. ${ }^{28}$ observaram que a prevalência de burnout era de 63,4\% considerando um escore alto do $\mathrm{MBI}$ e de 7,4\% considerando escore alto nas três dimensões, enquanto Tucunduva et al..$^{29}$ observaram em seu estudo prevalência de 52,3\%, segundo os critérios de Grunfeld et al., e de 3\%, segundo os critérios de Ramirez et al., dados esses que vêm corroborar os nossos achados.

A prevalência da síndrome com escore alto em cada uma das três dimensões analisadas foi de 35\% na exaustão emocional, 6,7\% na despersonalização e 25\% na redução da realização pessoal, achados esses parecidos com o de Tironi et al. ${ }^{14}$.

A principal dimensão afetada entre os médicos avaliados foi a exaustão emocional (35\%), considerada a dimensão precursora da síndrome ${ }^{8}$. Uma vez exaustas, as pessoas sentem cansaço físico e emocional, com dificuldade de relaxar, levando à redução dos recursos internos para enfrentar as situações vivenciadas no trabalho, bem como da energia para desempenhar as atividades ${ }^{7,12}$.

Diante dos sintomas psicológicos e físicos, o profissional desenvolve a despersonalização, caracterizada por atitudes distantes e negativas, ocorrendo um tratamento depreciativo das pessoas diretamente envolvidas com o trabalho ${ }^{26}$. Em nosso estudo, foi baixa a prevalência dessa dimensão $(6,7 \%)$.

Uma vez que o profissional se sente ineficiente, com diminuição da autoconfiança e sensação de fracasso, há redução na realização pessoal no trabalho, tida por muitos autores como a última reação ao estresse gerado pelas exigências ocupacionais ${ }^{12,30}$. A redução da realização profissional foi observada em 25\% dos médicos avaliados.

Considerando o critério de Grunfeld et al., encontramos associação estatisticamente significante com maiores níveis da síndrome entre sexo feminino ( $p=0,0304)$, trabalho em UTI adulto ( $p=0,0004)$, trabalhar em mais de dois hospitais ( $p=0,0095)$ e número de pacientes maior que 10 por plantão ( $p=0,0021)$, achados similares aos da literatura12,16,21,26,28.

Estudos revelam que há uma relação entre burnout e o sexo feminino 6,9,12,31-33, provavelmente devido à dupla jornada de trabalho (a profissional e a do lar) a que a maioria está sujeita9 ${ }^{9}$ e que há diminuição significante da síndrome nas profissionais que recebem apoio familiar ou de amigos ${ }^{33}$.

Em relação ao tipo de UTI, o tipo adulto apresenta maior prevalência da síndrome de burnout provavelmente devido à maior quantidade de pacientes assistidos. Uma das principais características para a ocorrência da síndrome é o contato direto com os pacientes ${ }^{9}$, portanto quanto maior a quantidade de pacientes assistidos e o número de hospitais em que o médico trabalha, maiores são as chances de o profissional desenvolver a síndrome de burnout ${ }^{12,14,26,28}$.

A literatura mostra que há maior incidência de burnout em profissionais com pouco tempo de atuação na área, devido à pouca experiência na profissão9,34, porém não houve associação em nosso estudo.

Apesar de não haver relação significativa do burnout com a idade em nosso estudo, a literatura descreve maior incidência da síndrome em profissionais com idade inferior a 30 anos, atribuída à pouca experiência, fator que desencadearia insegurança e, consequentemente, estresse ocupacional9,12.

Vários estudos atribuem ao casamento, ou ao fato de ter um companheiro estável, menor propensão ao burnout 1,12,28, entretanto verificou-se maior prevalência no presente estudo em médicos casados. Embora o poder estatístico de análise seja limitado pelo tamanho amostral reduzido, uma hipótese possível para esse achado é a de que características ligadas a resiliência e mecanismos psicológicos de enfrentamento do estresse (coping) podem variar entre os indivíduos deste grupo, sobrepujando eventuais fatores de proteção.

Em relação às especialidades médicas e, consequentemente, ao tipo de UTI, estudos revelam que em UTIs neonatal as alterações psicológicas eram prevalentes em 27\% dos médicos ${ }^{32,33}$, enquanto nas UTIs adulto a prevalência ficaram em torno de 63,4\% ${ }^{14,26,28}$, resultados semelhantes aos obtidos.

A longa jornada de trabalho é identificada como um dos agentes estressores da profissão médica . Porém, em nosso estudo não se observou relação significativa entre a carga horária do trabalho médico e a síndrome de burnout.

O presente estudo consiste em uma pesquisa transversal, que reflete a realidade pontual, podendo ser uma limitação do trabalho, no entanto destaca-se que o objeto de pesquisa foi investigado com diversas variáveis de estudo. Desse modo, contribui-se ao identificar que o ambiente da UTI adulto pode ser um fator de risco para o desenvolvimento da síndrome de burnout, enquanto a assistência em UTI com menos de dez leitos e menor quantidade de hospitais em que o profissional trabalha são fatores protetores.

\section{CONCLUSÃO}

O perfil dos médicos estudados é de uma população jovem, predominantemente feminina, casada, com menos de dez anos de formado, tendo como principal especialidade a Neonatologia, trabalhando há menos de cinco anos na UTI, 
em cerca de dois hospitais, cuidando de seis a dez pacientes por plantão e com elevada carga horária de trabalho.

Os principais fatores estressantes em UTI foram os ruídos excessivos e a possibilidade de complicações aos pacientes internados, fatores esses que podem contribuir para o surgimento da síndrome.

Os resultados apontaram elevada prevalência da síndrome de burnout nos médicos plantonistas, associada principalmente ao sexo feminino, trabalho em UTI adulto, trabaIhar em mais de dois hospitais e número de pacientes maior que dez por plantão. O reconhecimento dos fatores estressores associados ao burnout pode orientar mudanças no ambiente do trabalho que promovam o aumento da qualidade dos cuidados médicos e a promoção da saúde mental entre os profissionais.

\section{CONTRIBUIÇÕES INDIVIDUAIS}

Flávia Lopes Carvalho - Participou da concepção e do desenho do estudo, da revisão de literatura, análise e interpretação dos dados e da elaboração do artigo e aprovação da versão final.

Gabriela Lopes Carvalho Marques - Participou da concepção e do desenho do estudo, da revisão de literatura, análise e interpretação dos dados e da elaboração do artigo e aprovação da versão final.

Gilberto Sousa Alves - Participou da revisão de literatura, análise e interpretação dos dados e da elaboração do artigo e aprovação da versão final.

Hamilton Raposo Miranda Filho - Participou da revisão de literatura, análise e interpretação dos dados e da elaboração do artigo.

Sandra Fortes - Participou da análise e interpretação dos dados e aprovação da versão final.

\section{CONFLITOS DE INTERESSE}

Os autores não possuem conflitos de interesse a serem declarados.

\section{AGRADECIMENTOS}

Agradecemos a todos os envolvidos nesta pesquisa, em especial Fernanda Ferreira Lopes, pelo auxílio prestado na análise e interpretação dos dados.

\section{REFERÊNCIAS}

1. Trigo TR, Teng CT, Hallak JEC. Síndrome de burnout ou estafa profissional e os transtornos psiquiátricos. Rev Psiquiatr Clín. 2007;34(5):223-33.
2. Dejours C. A loucura do trabalho. 5 a ed. São Paulo: Cortez/Oboré; 1992. p. 168.

3. Fogaça MC, Carvalho WB, Nogueira, Martins LA. Estudo preliminar sobre a qualidade de vida de médicos e enfermeiros intensivistas pediátricos e neonatais. Rev Esc Enferm USP. 2010;44(3):708-12.

4. Vieira LC, Guimarães LAM, Martins DA. 0 estresse ocupacional em enfermeiros. In: Guimarães LAM, Grubits S. Saúde mental e trabalho. 3a ed. São Paulo: Casa do Psicólogo; 2003. p. 169-85.

5. Gracino ME, Zitta ALL, Mangili OC, Massuda EM. A saúde física e mental do profissional médico: uma revisão sistemática. Saúde Debate. 2016;40(110):244-63.

6. Lima RAS, Souza Al, Galindo RH, Feliciano KVO. Vulnerabilidade ao burnout entre médicos de hospital público do Recife. Ciênc Saúde Coletiva. 2013;18(4):1051-8.

7. Schaufeli WB, Buunk BP. Burnout: an overview of 25 years of research and theorizing. In: Schabracq MJ, Winnubst JAM, Cooper CL. The handbook of work and health psychology. 2a ed. New York: Wiley; 2003. p. 383-425.

8. Maslach CA. Multidimensional theory of burnout. In: Cooper CL, editor. Theories of organizational stress. Oxford: Oxford University Press; 1998. p. 68-85.

9. Benevides-Pereira AMT, editor. Burnout: quando o trabalho ameaça o bem-estar do trabaIhador. 4a ed. São Paulo: Casa do Psicólogo; 2002.

10. Rubow B. Stress and burnout in the teaching profession: European studies, issues, and research perspectives. In: Vanderbergue R, Huberman A, editors. Understanding and preventing teacher burnout: a source book of international practice and research. Cambridge: Cambridge University Press; 1999. p. 38-58.

11. França HH. A síndrome de "burnout". Rev Bras Med. 1987;44(8):197-9.

12. Maslach C, Schaufeli WB, Leiter MP. Job burnout. Annu Rev Psychol. 2001;52:397-422.

13. Maslach CP, Jackson SE. The measurement of experienced burnout. J Occup Behav. 1981;2(2):99-113.

14. Tironi MOS, Nascimento Sobrinho CL, Barros DS, Reis EJFB, Marques Filho ES, Almeida A, et al. Trabalho e síndrome da estafa profissional (síndrome de burnout) em médicos intensivistas de Salvador. Rev Assoc Med Bras. 2009;55(6):656-62.

15. Barros MMS, Almeida SP, Barreto ALP, Faro SRS, Araújo MRM, Faro A. Síndrome de Burnout em médicos intensivistas: estudo em UTIs de Sergipe. Temas Psicol. 2016;24(1):377-89.

16. Tironi MOS, Teles JMM, Barros DS, Vieira DFVB, Silva Filho CM, Martins Júnior DF, et al. Prevalência de síndrome de burnout em médicos intensivistas de cinco capitais brasileiras. Rev Bras Ter Intensiva. 2016;28(3):270-7.

17. Guntupalli KK, Fromm RE Jr. Burnout in the internist - intensivist. Intensive Care Med. 1996;22(7):625-30.

18. Friedman G, Vieira RF. Alta prevalência de síndrome de burnout em médicos intensivistas da cidade de Porto Alegre. Rev Bras Ter Intensiva. 2017;29(1):115-20.

19. Monesterolo F, Toledo J, Moreno A, Vargas S, Orland MC. Síndrome de burnout en médicos de terapias intensivas de General Roca y Cipolletti. Rev Arg Ter Intensiva. 2015;32(4).

20. Nassar Junior AP, Azevedo LCP. Fatores associados à satisfação profissional e pessoal em intensivistas adultos brasileiros. Rev Bras Ter Intensiva. 2016;28(2):107-13.

21. World Health Organization. Guidelines for the primary prevention of mental, neurological and psychosocial disorders: staff burnout. Geneva: Division of Mental Health World Health Organization; 1998. p. 91-110.

22. Pereda-Torales L, Celedonio FGM, Vásquez MTH, Zamora MIY. Sindrome de burnout en médicos y personal paramédico. Salud Mental. 2009;32(5):399-404.

23. Carlotto MS, Camara SG. Propriedades psicométricas do Maslach Burnout Inventory em uma amostra multifuncional. Estud Psicol. 2007;24(3):325-32.

24. Ramirez AJ, Graham J, Richards M, Cull A, Gregory WM. Mental health of hospital consultants: the effects of stress and satisfaction at work. Lancet. 1996;347(9003):724-8.

25. Grunfeld E, Whelan TJ, Zitzelsberger L, Willan AR, Montesanto B, Evans WK. Cancer care workers in Ontario: prevalence of burnout, job stress and satisfaction. CMAJ. 2000; $163(2): 166-9$.

26. Nascimento Sobrinho C, Barros DS, Tironi MOS, Marques Filho ES. Médicos de UTI: prevalência da síndrome de burnout, características sociodemográficas e condiçōes de trabalho. Rev Bras Educ Med. 2010;34(1):106-15. 
27. Lacerda JC, Barbosa AP, Cunha AJLA. Perfil profissional do intensivista pediátrico no estado do Rio de Janeiro, sudeste do Brasil. Rev Bras Ter Intensiva. 2011;23(4):462-9

28. Barros DS, Tirone MOS, Nascimento Sobrinho CL, Neves FS, Bittencourt AGV, Almeida AM, et al. Médicos plantonistas de unidade de terapia intensiva: perfil sócio-demográfico, condições de trabalho e fatores associados à síndrome de burnout. Rev Bras Ter Intensiva. 2008;20(3):235-40.

29. Tucunduva LTCM, Garcia AP, Prudente FVB, Centofanti G, Souza CM, Monteiro TA, et al. A síndrome da estafa profissional em médicos cancerologistas brasileiros. Rev Assoc Med Bras. 2006;52(2):108-12.

30. Lima FD. Características da incidência da síndrome de burnout em pediatras de uma organização hospitalar pública [dissertação]. Florianópolis: Programa de Pós-Graduação em Psicologia do Centro de Filosofia e Ciências Humanas da Universidade Federal de Santa Catarina; 2004.
31. Lautert L. 0 desgaste profissional: uma revisão da literatura e implicações para a enfermeira. Rev Gaúcha Enferm. 1997;18(2):83-93.

32. Oates PR, Oates RK. Stress and work relationships in the neonatal intensive care unit: are they worse than in the wards? J Paediatr Child Health. 1996;32(1):57-9.

33. Oates RK, Oates P. Stress and mental health in neonatal intensive care units. Arch Dis Child Fetal Neonatal Ed. 1995;72(2):107-10.

34. Dyrbye LN, West CP, Satele D, Boone S, Tan L, Sloan J, et al. Burnout among U.S. medical students, residents, and early career physicians relative to the general U.S. population. Acad Med. 2014;89(3):443-51. 